\title{
DRIFT OF SUNSPOTS IN LATITUDE
}

\author{
JAAKKO TUOMINEN \\ University of Helsinki, Finland
}

I am going to show two slides which should clearly prove that sunspots are drifting very regularly in the north-south direction on the solar surface.

Slide 1 is from a paper published in 1941. On the $x$-axis is the heliographic latitude. The $y$-axis gives the average proper motion of sunspots in latitude, expressed in degrees per day. The material is composed of long-lived spots, i.e. spots which have been observed at least at two rotations of the Sun. The dotted curve gives northern and southern latitude spots treated separately. The continuous curve has been drawn using averages of the values on the northern and southern latitudes. The diagram shows clearly that, on the average, between the parallels $\pm 16^{\circ}$ sunspots are drifting towards the equator, while outside these parallels they are drifting towards the poles.

In the year 1954 Udo Becker showed that the drift curve changes when spots around sunspot minima and maxima are separated. And what is interesting, the errors decrease from the values obtained when all spots are combined. This happens in spite of the fact that in the separated material the number of values is much smaller than in the case when all the spots are combined. It clearly proves that the drift curve around minimum is different from that around maximum.

Slide 2. In this slide the dotted curve represents the values derived by Becker. Here the latitude is on the $y$-axis and the mean daily proper motion in latitude on the $x$-axis. Becker's material was practically the same as that of the first slide, i.e. long-lived spots only. As the number of spots in the minimum phase is small compared to that in the maximum phase, the curve of Slide 1 practically corresponds to the curve of the maximum phase, which can be seen easily.

Unfortunately, Becker did not treat northern and southern latitudes separately, but combined the two hemispheres. But, in order to obtain a check of the result, in 1960 I made an exactly similar study to that of Becker, this time based entirely on short-lived spots. Then the continuous line on the slide was obtained. Its accuracy is not inferior to that obtained from long-lived spots, because the number of short-lived spots is much greater than that of long-lived ones. It is seen that the two entirely different materials give practically the same result. It can be asked why there is such an oscillation in the north-south drift of sunspots.

\section{References}

Becker, U. 1954, Z. Astrophys. 34, 129.

Tuominen, J.: 1941, $Z$. Astrophys. 30, 96.

Tuominen, J.: 1960, Z. Astrophys. 51, 91. 